\title{
Transformación de la familia en Colombia: mujer e infancia
} (1968-1984)

Transformation of the family in Colombia: women and children

(1968-1984)

Por: Absalón Jiménez Becerra*

\section{Resumen:}

La familia, escenario institucional para la materialización de la experiencia infantil, se transformó en las últimas décadas del siglo XX debido al desarrollo de la farmacología y la biotecnología - disciplinas pioneras de la planificación familiar. Con respecto a Colombia, en dicho lapso surgieron una serie de iniciativas que tuvieron como fin la protección de la familia; en 1968, por ejemplo, se fundó el Icbf (Instituto Colombiano de Binestar Familiar). La protección de la familia es sinónimo de la protección de la infancia. Ésta es objeto de una serie de prácticas y discursos inmanentes al cuerpo infantil, los cuales pretenden ser indagados por medio de la presente investigación.

\section{Palabras claves:}

Familia, infancia, juego, socialización.

\begin{abstract}
:
The family as the primary institutional setting for the realization of children's experience becomes the last decades of the 20th century, the development of Pharmacology and biotechnology, in which family planning takes body product. In the Colombian area in the period to study are a series of initiatives designed to the protection of the family in 1968 was born the ICBF. The protection of the family is the protection of children, which ends up being subject to a number of practices and immanent to the child body speeches which seek to be indagados in this research.
\end{abstract}

\section{Key words:}

Family, children, game, socialization.

* Absalón Jiménez Becerra - abjibe@hotmail.com. Universidad Distrital Francisco José de Caldas. 


\section{Introducción}

La familia es una institución occidental originada en la modernidad y consolidada a lo largo de los siglos XVII y XVIII. Como lo demostró Philippe Aries (1987), la familia, más que una institución, es un sentimiento ligado a la infancia. En términos espaciales, si se tiene en cuenta la sociedad de la aldea antigua, la familia demandó un mínimo de privacidad y aislamiento con la instauración de la casa, lugar en el cual empezó a acentuarse una revolución de carácter afectivo tan profunda entre sus miembros que trascendió, incluso, el momento de la muerte (p. 482). De hecho, la muerte de nuestros abuelos y padres será recordada hasta la vejez propia. La familia y la casa —como expresión de lo privado - fueron transformándose paulatinamente hasta consolidar la esfera de lo íntimo; décadas después, las construcciones de las casas se dividieron mediante un corredor y los hijos fueron trasladados del cuarto de sus padres a habitaciones independientes (separados de acuerdo a su sexo). La infancia, entonces, se convirtió en el centro de la familia moderna y sobre ella empezó a girar buena parte de la dinámica familiar. La niñez moderna demandó una propuesta educativa y socializadora que se ubicabó más allá de la frontera familiar. Tiempo después sería complementada por la escuela.

Entre tanto, Norbert Elias (1998) ubica las discusiones en torno a la familia y la infancia dentro del marco del proceso civilizatorio vivido en Occidente. Pensar a los niños en la modernidad es darse cuenta de su relativa autonomía, pues se van haciendo adultos individualmente a lo largo de un proceso civilizador que varía acorde al estado del desarrollo de los respectivos modelos sociales (p. 418). La reflexión más profunda acerca de las necesidades y características de los niños es, en el fondo, el reconocimiento a su derecho de ser comprendidos y apreciados bajo su carácter propio. Aunque existen todavía una serie de relaciones de poder tradicionales en el interior de la familia, los niños, en la sociedad moderna y contemporánea, también ejercen un poder sobre los padres. En términos sociológicos, la autonomía del niño — como principal característica civilizatoria-estableció su reconocimiento como sujeto tanto en el interior de la familia como en el de la sociedad.

La familia moderna, base fundamental en la constitución de los Estado-nación, entraría en crisis hacia la segunda mitad del siglo XX (en el tránsito de la sociedad disciplinaria a la sociedad de control $\left.^{1}\right)$. Particularmente, para la coyuntura de 1960, se vería amenazada por varias razones: la incorporación masiva de las mujeres en el trabajo remunerado, la trasformación tecnológica, el desarrollo de la biología y la farmacología, la generación del dispositivo y la píldora anticonceptiva, el movimiento feminista, el cambio de las relaciones heterosexuales y las ideas de la globalización cultural (Castells, 1999, p. 159). Para sociólogos como Manuel Castells, la crisis de la familia patriarcal ha traído como consecuencia la transformación de la identidad sexual y de los sistemas de personalidad. En las generaciones actuales es evidente la constitución de una nueva revolución sexual en la que la relación de pareja procreativa y tradicional (relacional y de compañía), que caracterizó el siglo XIX y la primera mitad del siglo XX, es reemplazada por otro tipo de sexualidad. El deseo por el cuerpo del otro se convirtió en sinónimo de transgresión y amenaza los valores tradicionales de la familia y el sentimiento de infancia moderno (centro de esta institución).

Por otra parte, Anthony Gidden (2004) coincide en el análisis; según él, la vida sexual en la actualidad se ha convertido en un proyecto personal abierto y constituyente de demandas y nuevas ansiedades, pues la mayoría de hombres y mujeres, antes

1 Michael Hard y Antonio Negri, Imperio (Barcelona, Paidós, 2002)En esta obra, desde una perspectiva foucultiana, se da a conocer cómo desde la década los sesenta se instaura de manera paulatina un nuevo orden mundial que incorpora la totalidad del globo. Este nuevo orden, liderado por la economía de consumo y los medios de comunicación, crea una nueva subjetividad constitutiva de los sujetos. Las nuevas formas de control ya están instaladas en los sitios de encierro como la escuela, la fábrica y la cárcel, y también se encuentran interiorizadas en el cuerpo y la mente de los sujetos. La instalación de la sociedad de control, vista como aquella sociedad que se desarrolla en el borde último de la modernidad, ha incidido de manera directa en la transformación de instituciones sociales modernas como la familia. 
del matrimonio, tienen una experiencia amplia en su vida sexual. Entonces, el amor romántico — característico de la modernidad-, mediante el cual se idealizaba una pareja, se establecía un hogar y se concebían a los hijos, al encontrarse en crisis, está siendo remplazado por el amor pasional y confluente (p. 43). Éstos, además de ubicar a la sexualidad como una definición progresiva del sexo, la incluye; el nuevo modelo de relaciones afectivas no es matrimonial sino que se fundamenta en términos de flexibilidad - demanda un disfrute del cuerpo. Para los jóvenes heterosexuales que establecen una relación no son prioridad ni el matrimonio ni los hijos, hecho que invita al cuestionamiento del sentimiento de familia e infancia moderno (está empezando a ser visto como tradicional e histórico).

Sin lugar a dudas, la trasformación de la familia en las últimas décadas es un punto de partida para reflexionar, a partir de disciplinas como la sociología, diversos aspectos referentes a los tipos de sentimientos que las consolida en la actualidad. Por su parte, la instauración institucional de la familia individualizó paulatinamente a los niños hasta reconocerlos como sujetos autónomos mediante la socialización.

Tomando como referencia el contexto descrito en las líneas anteriores, el objetivo de este trabajo es evidenciar, dentro de la actual institución familiar colombiana, las condiciones de emergencia y las procedencias de la infancia. Para ello, la herramienta metodológica a implementar fue la consulta de fuentes primarias con cierto grado de prioridad hacia el diario El Tiempo - debido a su cobertura nacional durante más de un siglo- en el lapso comprendido entre 1968 y 1984. Éste primer rastreo permitió establecer las aristas de la discusión y decantar la temática de la perspectiva epistemológica con el fin de dar a conocer el resultado de una perspectiva analítica en la que las fuentes son, en buena medida, las que reconstruyen un discurso que delimita la infancia en esta coyuntura.

En este sentido, según los lineamientos establecidos por Michael Foucault en torno a la proposición de la infancia vista como sujeto, nos situamos, en este primer avance, en las instituciones y no en las discusiones (la familia colombiana después de la segunda mitad del siglo XX). De tal manera, "el sujeto es el producto de una experiencia institucional y la obligación del investigador es explicar su aparición y su existencia por medio de unas prácticas sociales y discursivas que paulatinamente lo rodean y lo delimitan" (Quiceno, 2007, p. 3). Por consiguiente, este ejercicio investigativo es la respuesta a una inquietud mediante la cual queremos dar cuenta de las infancias recientes - aún presentes en la memoria individual y colectiva- que vivieron un proceso particular de naturalización y socialización y que facilitaron la procedencia de la infancia actual, reconocida como un sujeto portador de subjetividad e identidad. Entonces, la idea puesta en escena es que la procedencia de la infancia contemporánea tiene un antecedente directo en la transformación de la familia; el nuevo rol de la mujer y el discurso de la socialización (etc.) son aspectos que delimitan y definen a la infancia actual.

La transición de la familia moderna a la familia contemporánea en Colombia debe entenderse de acuerdo a unas condiciones de emergencia establecidas por Occidente - a mediados de la década de los sesenta - en las que se logró separar la vida reproductiva de la mujer del placer sexual. Dicha situación posibilitó, en nuestro país, la reestructuración social de la familia y así la mujer empezó a ejercer un rol nuevo. Las prácticas de crianza, por su parte, iniciaron un proceso de modernización tomando como ejemplo el mundo occidental desarrollado.

La individualización y delimitación de la niñez en las prácticas discursivas, tanto sociales como académicas y disciplinares, evidencian un punto importante en su reconocimiento, así como el interés por controlar éste nuevo sujeto (primera mitad de los años ochenta). Es en ésta coyuntura de corte donde se cierra el primer ejercicio investigativo y en la que se dan a conocer las condiciones de emergencia de la infancia colombiana contemporánea a partir de la perspectiva familiar. 


\section{Consideraciones metodológicas}

Respecto al problema de investigación es fundamental dar a conocer nuestro "dispositivo de análisis"2, el cual permite desarrollar un balance primario de las condiciones de emergencia en la infancia contemporánea. En buena medida, el propósito de este trabajo consiste en indagar la forma en la cual la familia se convierte en un elemento consustancial del dispositivo que delimita la nueva infancia.

En este sentido, desde cierta perspectiva inspirada en Foucault, el dispositivo "es lo que organiza, distribuye, distingue o reúne elementos, lo que vuelve inteligible un conjunto confuso, lo que indica para el pensamiento las orientaciones de la investigación. Nos permite delimitar, ver de qué manera las cosas y las personas se disponen. Por lo demás, es una herramienta dinámica, variable y adaptable a situaciones siempre cambiantes" (1976) ${ }^{3}$. Entonces el dispositivo puede responder a una cartografía en la que se dibuje un mapa donde serán localizadas las condiciones de emergencia de la infancia actual. Para tal efecto, el mecanismo propuesto debe situarse en un contexto histórico en particular acompañado de una serie de $<<$ emplazamientos de sujeto $>>^{4}$, así como de posiciones e instituciones

2 El presente dispositivo se inspira en la iniciativa metodológica desarrollada por Michael Foucault (1976), Historia de la sexualidad. La voluntad de saber, tomo I, México D.F., Siglo XXI; para abordar el tema de la sexualidad en la modernidad plantea cuatro puntos: la apuesta, el método, el dominio y la periodización.

3 En este sentido, son aclaratorias a la visión de dispositivo de Rene Schérer (2005): “A su disposición”, en: Foucault, la Pedagogía y la Educación. Pensar de otro modo, Bogotá, Magisterio, Grupo Historia de las Prácticas Pedagógicas.

4 Para cada enunciado existen emplazamientos de sujeto muy variables y particulares. En cada enunciado debemos distinguir tres círculos como tres posiciones de espacio. Primero, un espacio colateral, asociado o adyacente, formado por otros enunciados que forman parte del mismo grupo. En este sentido, debemos aclarar que el enunciado no es lateral ni vertical; es trasversal y se encuentran en un mismo nivel, que convierten a la familia como tal en un medio de dispersión y heterogeneidad, justo contrario a la homogeneidad. Cada enunciado es inseparable de una multiplicidad rara y regular a un tiempo. Bajo este principio, cada enunciado es multiplicidad, y no una estructura o un sistema. La segunda porción de espacio es el correlativo: en éste se trata de la relación del enunciado, ya en las cuales se materialicen prácticas sociales soportadas con discursos que traten sobre la actualidad de la infancia.

El dispositivo de análisis histórico propuesto, aún cuando puede ser mucho más amplio en esta investigación, procura dar cuenta del paso de la infancia moderna a la contemporánea desde la institución familiar — base fundamental del proceso de naturalización, socialización y subjetivación de este sujeto. Así pues, la familia cumple un papel fundamental ya que se desplaza desde una perspectiva epistemológica y metodológica particular (determina las prácticas sociales y discursivas constitutivas del niño en un periodo determinado).

Por lo tanto, este texto se mueve en un frente doble: el genealógico y el arqueológico. En el primero busca:

Los comienzos innombrables que dejan esa sospecha de color, el análisis de procedencia que permita disociar al Yo y hacer pulular en los lugares y plazas de su síntesis vacía, mil sucesos perdidos hasta ahora. La pregunta por la procedencia de la infancia contemporánea en Colombia permite encontrar, bajo el aspecto único de un carácter $\longrightarrow$ de un concepto-, la proliferación de los sucesos mediante los cuales se ha formado. La genealogía no pretende remontar el tiempo para restablecer una gran continuidad por encima de la dispersión del olvido sino continuar la filial compleja de la procedencia, mantener lo que pasó en la dispersión que le es propia, es percibir los accidentes, las desviaciones o los retornos

no con otros enunciados, sino con los sujetos, sus objetos, sus conceptos. Un mismo enunciado puede tener varias posiciones, varios emplazamientos de sujeto; un autor o un narrador o bien un firmante a un autor de una carta. En consecuencia, el Yo, deriva del enunciado, "del habla" o "se habla". La tercera posición de espacio es extrínseco: el espacio complementario o de formaciones no discursivas. Para Foucault, una institución implica enunciado. Y a la inversa, los enunciados remiten a un medio institucional, sin el cual no podrían formarse ni los objetos que surgen en tales localizaciones del enunciado, ni el sujeto que habla desde tal emplazamiento. Consultar el capítulo "El nuevo archivista” en Guilles Deleuze, Foucault, Barcelona, Paidós (1987). 
completos. La búsqueda de procedencia no funda, al contrario, remueve aquello que se percibía inmóvil, fragmenta lo que se pensaba unido; muestra la heterogeneidad de aquello que se imaginaba conforme así mismo. La genealogía como el análisis de procedencia, se encuentra por tanto en la articulación del cuerpo y de la historia. Debe mostrar al cuerpo impregnado de historia, y a la historia como destructor del cuerpo (Foucault, 1991, pp. 12-15).

En consecuencia, la historia de un saber "representan desde la perspectiva de Foucault una posibilidad tanto de análisis arqueológico con orientación epistemológica de análisis genealógico que puede dar cuenta de la formación de objetos, conceptos y técnicas en la interioridad de las prácticas sociales" (Zuluaga, 1999, p. 5). Para Olga Lucía Zuluaga el saber - en este caso sobre la infancia - configura un dominio que se puede historiar con herramientas tanto arqueológicas como de la genealogía por medio de la cual se busca dar a conocer "desde la inspección del archivo una estrategia de análisis, que designa el tema general de una descripción que interroga lo ya dicho a nivel de su existencia, de la función enunciativa que se ejerce en él, de la formación discursiva a que pertenece, del sistema general de archivo de que depende" (p. 35).

De acuerdo a los análisis de las fuentes primarias, el núcleo familiar colombiano se transformó y se modernizó a finales de la década de los sesenta, abriendo el espacio para lo que posteriormente sería la modernización de la familia y, en consecuencia, el paso definitivo de la infancia moderna a la contemporánea.

Para tal efecto he abordado las transformaciones de la familia colombiana en la segunda mitad de los años sesenta; la discusión fue retomada como producto de los cambios que se vivían en Occidente. Colombia, en ese entonces, experimentó la polarización de varios temas controversiales: discusiones de carácter global, métodos de planificación y el uso de la píldora anticonceptiva; entre otros, generaron una discusión inicial en torno al tema de la familia y al nuevo rol de la mujer. Las implicaciones posteriores de ésta transformación tuvieron consecuencias en las prácticas de crianza; buscaron moldear y adaptar a los niños hacia el capitalismo.

\section{La planificación familiar}

La controversia generada por el uso de la píldora — entre la segunda mitad de los años sesenta y la primera de los setenta - llamó la atención en varios focos sociales de Occidente. Por su parte, en el escenario colombiano, la Iglesia Católica sustentó que el implemento de éste fármaco amenazaría la estabilidad de la familia tradicional por dos razones fundamentales: los nuevos métodos de planificación y la incorporación de la mujer al trabajo remunerado. Así mismo, ésta situación supuso dos pronunciamientos; uno del Episcopado Colombiano y otro de la encíclica Humanae Vitae (agosto de 1968) basados en la libertad del número de hijos en las familias católicas y en la oposición a los nuevos métodos de planificación.

Para algunos especialistas de la época no fue casualidad que la familia empezara a ocupar un lugar preponderante dentro de las preocupaciones del mundo moderno. En ese sentido, "los Estados comienzan a salir de un inexplicable letargo con relación a los problemas por los cuales atraviesa la familia. No importa el tinte ideológico de las legislaciones o su distribución geográfica en el mundo. Entre nosotros se inician esfuerzos serios por tutelar esta célula básica de la sociedad" (El Tiempo, 1968, 3 de mayo, p. 5).

Entre tanto, la familia tradicional se transformó también en su exterior, pues la biotecnología inició un desarrollo a pasos agigantados a pesar de la oposición de la Iglesia (su poder en el establecimiento de la moral en los núcleos familiares comenzó a verse amenazado).

Bajo esa preocupación, el Secretario Permanente del Episcopado aclaró, en una declaración pronun-

5 "La familia: el diálogo... una receta". El Tiempo, 3 de mayo de 1968 , p. 5.

REVISTA CIENTÍFICA / ENERO -DICIEMBRE DE 2010 / No. 12 / BOGOTÁ, D.C. 
ciada el 15 de junio de 1968, que los únicos seres con el poder de decidir el número de los hijos eran los padres. Además, cuestionó la ayuda financiera de las grandes potencias porque "no debe acompañarse de presiones a favor de la limitación de nacimientos. Deploramos que la ayuda en alimentos o en dinero esté sometida a condiciones expresas o no, que implican la restricción de los nacimientos. Nuestro país no puede permitirse el imponer los puntos de vista de los demás, ya se trate de su desarrollo o el número de hijos" (El Tiempo, 1968, 15 de junio, p. 30) ${ }^{6}$.

En ésta misma coyuntura, el Papa Pablo VI prohibió el uso de medios artificiales de concepción para más de 500 millones de católicos y pidió a todos los gobiernos del mundo el veto de tales procedimientos "degradantes" (El Tiempo, 1938, 30 de julio, p. 1). El pronunciamiento del Papa sobre el control de la natalidad significó el epílogo de una controversia acentuada en 1963, cuando la explosión poblacional inquietó a los gobiernos y la opinión pública se dividió. La encíclica Humanae Vitae de agosto de 1968 generó discrepancias y desconciertos en el ámbito nacional al considerar el método rítmico - sistema Ogino-Knaus - como el único aprobado por la Iglesia Católica. Para los creyentes, la abstención sexual mediada por el método del ritmo era algo contradictorio, pues condenaba a los infortunados cónyuges católicos a practicar un tipo particular de castidad en el interior del hogar.

Amparo Gómez, especialista de la época en el tema familiar, manifestó su comprensión frente a la posición de la Iglesia debido al posible desenfreno que en las jóvenes solteras pudiera traer el uso y el abuso de las píldoras:

Con las restricciones que ha impuesto el Papa sólo logrará crear un gran complejo de culpa en los cónyuges católicos que no puedan correr el riesgo del método Ogino-Knauss, sin que falten contra la responsabilidad paterna. Es muy contradictoria

6 "Se censura intervención en control de natalidad". El Tiempo, 15 de junio de 1968, p. 30.

7 "Pablo VI condena control natal". El Tiempo, 30 de julio de 1968, p. 1. la actitud de la Iglesia, pues mientras acepta la necesidad de limitar el número de hijos no permite los métodos seguros y adecuados a cada pareja... Llegará el momento en el cual tendremos que escoger entre vivir en el pecado o padecer un pequeño infierno en vida." (El Tiempo, 1968, 5 de agosto, p. 13) ${ }^{8}$

Años atrás, la discusión acerca de la píldora anticonceptiva había estremecido a la sociedad pero fue hasta 1968 cuando se convirtió en la protagonista mundial de una controversia entre la Iglesia Católica y los Estados modernos; fue considerada un problema biológico y social. Entre tanto, la encíclica Humana e Vitae, fundamentada en el "método del ritmo" como el reconocido por la Iglesia, decretó - para las parejas católicas - que la única forma posible para regular el tamaño de la prole era confiando las relaciones sexuales al periodo femenino ${ }^{9}$ (El Tiempo, 1968, 4 de agosto, p. 16). La nueva encíclica, al condenar la esterilización, el aborto y la píldora — situándolas en el mismo nivel- abrió un camino tentativo hacia la infidelidad matrimonial, la inmoralidad, la pérdida del respeto a la mujer e, inclusive, peligros de orden político.

A comienzos de la década del setenta, la Unicef, mediante numerosas campañas, distribuyó píldoras anticonceptivas a los gobiernos que las solicitaron. Ésta iniciativa polémica figura en el informe general del Director Ejecutivo de dicho organismo, Henry B. Labouse ${ }^{10}$ (El Tiempo, 1970, 1 de mayo, p. 10). En Colombia, para mayo de 1970, Profamilia dio a conocer importantes resultados en los programas de planificación familiar en Bogotá D.C.; 84.440 mujeres estaban planificando su familia por medio de métodos científicos. Ésta medida determinó, además de la reducción en las cifras del crecimiento poblacional, la rebaja de un número considerable de abortos - aspecto que, especialmente en los niveles socioeconómicos bajos, evidenció un

8 "Los católicos ante un dilema". El Tiempo, 5 de agosto, de 1968, p. 13.

9 "Estupor mundial por rechazo a anticonceptivos". El Tiempo, 4 de agosto de 1968, p. 16.

10 “UNICEF, dará píldoras como ayuda”. El Tiempo, 1 de mayo de 1970, p. 10. 
cambio de actitud general hacia la planificación familiar.

En consecuencia, en la Colombia de comienzos de los años setenta, la planificación familiar comenzó a ser debatida en todos los medios y niveles sociales sin que nadie se sintiera avergonzado. Profamilia, entidad creada en 1966 como una iniciativa de carácter no gubernamental — sin ánimo de lucropor parte de un grupo de médicos preocupados por el crecimiento demográfico, fue nombrada en 1968 como miembro de la Federación Internacional de Planificación Familiar. Por su parte, un par de años después, uno de sus fundadores, el ginecólogo Fernando Tamayo, estableció la planificación familiar en Colombia como una prioridad social. Para ese entonces, Colombia disputó con Chile el primer puesto en Hispanoamérica en lo referente a la aplicación de políticas de planificación familiar.

Según Profamilia, en la Bogotá de 1970:

El $42 \%$ de la población femenina se encuentra en el programa de planificación familiar, lo que ha significado un descenso importante en el movimiento de hospitales de maternidad, cuya cifra anual de partos ha permanecido estable en los últimos seis años lo que indica un descenso en la natalidad. En 1969 el número de pacientes nuevos para el programa superó en más del 100\% al de 1968 y el número de controles fue superado en un $100 \%$. Los casos nuevos en 1969 fueron 41.632 y los controles llegaron a 111.056. Para 1970, Profamilia registra que es muy importante el hecho de que la asociación fue invitada a dictar cursillos destinados a grupos de sacerdotes y seminaristas católicos y a través de los cuales se observó cómo los conceptos y actitudes de los eclesiásticos van cambiando a medida que ellos advierten una información técnica e imparcial sobre los problemas familiares y de población y sobre la planificación familiar ${ }^{11}$ (El Tiempo, 1979, 21 de mayo, p. 7).

11 "Planificación familiar en Bogotá, 84.480 mujeres". El Tiempo, 21 de mayo de 1970, p. 7
Aunque la píldora se convirtió en la mejor opción para la planificación familiar, en la Encuesta Nacional de Fecundidad, realizada entre marzo y agosto de 1969 y aplicada a 6.000 mujeres colombianas, se evidenció que el desconocimiento de la población frente a las bondades de dicho producto era bastante amplio. Por ejemplo, en las zonas rurales, el 55\% de las mujeres desconocía la existencia de la píldora; sin embargo, a nivel nacional, el método anticonceptivo menos ignorado fue, de manera paradójica, el uso de la píldora (lo conocían más de la mitad de las mujeres encuestadas). En segundo lugar se ubicó el ritmo, seguido de otros métodos como el preservativo, el coito interrumpido y la esterilización.

Para los especialistas, lo más grave de la píldora no era sólo que había que conocerla; sino conocerla a tiempo, pues la mayoría de las colombianas supieron de éste método cuando ya estaban casadas. Además, en el mismo estudio se dieron a conocer las diferencias existentes frente a los temas de planificación familiar entre el mundo urbano, el rural y las diversas clases sociales. La encuesta, que estuvo acompañada por un cuestionario de fertilidad, demostró la inclinación favorable hacia la limitación del número de hijos. Para la época, la mayoría de mujeres colombianas de 28 años de edad había tenido cinco embarazos ( 3 de ellos dados a luz) y contaban con 20 años más de fertilidad ${ }^{12}$.

En 1972, la tasa de crecimiento poblacional se convirtió en una preocupación de carácter público para el Gobierno colombiano. En ésta coyuntura, se impulsaron iniciativas concretas frente al control natal con el fin de detener la explosión demográfica y reducir dicho crecimiento que oscilaba entre el $3.4 \%$ y el $3.8 \%$ anual. De acuerdo con los estudios de la época, Colombia, entre un total de 142 naciones, era uno de los países con mayor tasa de crecimiento; lo superaban Costa Rica (3.8\%) y Kuwait — con un porcentaje similar. Entonces, según este ritmo de crecimiento, para 1990 Colombia sería un país con 45 millones de

12 "Control natal, medio para atacar el aborto". El Tiempo, 9 de diciembre de 1970, p. 7.

REVISTA CIENTÍFICA / ENERO -DICIEMBRE DE 2010 / No. 12 / BOGOTÁ, D.C. 
habitantes, aspecto que debía ser controlado por medio de campañas de planificación para evitar un desborde en la política social.

Frente a éste problema, el Gobierno propuso varias medidas para su solución: reducir la ilegitimidad en la constitución de la pareja mediante programas educativos promoviendo, a la vez, la responsabilidad ante los hijos; lograr una mejor distribución territorial de la población; contribuir a la disminución de la taza de morbilidad y mortalidad infantil; modificar el ritmo de crecimiento de la población y promover la información objetiva y adecuada referente a la vida familiar y sexual que permita tomar una decisión libre a la pareja ${ }^{13}$ (El Tiempo, 1972, 1 de febrero, p. 14).

Sin lugar a dudas, la planificación familiar, el volumen de la familia y la ilegitimidad de la pareja; entre otros, se habían convertido en una preocupación pública objeto de un discurso que intentó dar cuenta de una nueva realidad colombiana. Considerando el contexto de los temas anteriores, el debate en torno al aborto en Occidente había llegado a un punto interesante. En 1967, Inglaterra admitió en su legislación la posibilidad del aborto. En Estados Unidos, en ese mismo año, se aprobó la medida, en California (1971) y en momentos en que los estados de Nueva York, Alaska y Hawai dieron luz verde al aborto en sus jurisdicciones. Por su parte, en Francia, según el Instituto Francés de Opinión Pública, el 55\% de sus habitantes aprobaban la legalización del aborto No obstante, en la sociedad occidental de los años setenta, el crecimiento demográfico y la sensibilidad ante los derechos de la madre eran mucho más considerables que en la época clásica.

En la primera mitad de la década del setenta, el ex presidente Carlos Lleras Restrepo, padre de la Ley de la Patria Potestad (Ley 75 de 1968), se convirtió en uno de los especialistas más reconocidos del tema familiar en Colombia. En junio de 1972, durante una de sus intervenciones, estableció las razones de carácter económico por las cuales el Estado

13 “Reducirán tasa natal". El Tiempo, 1 de febrero de 1972, p. 14. debía apoyar tanto los programas de planificación familiar como su incidencia en la política social:

El Gobierno al tratar de elevar el ingreso per cápita, no le basta con modificar la política que opera sobre los agregados económicos. Debe tenerse en cuenta que existe la posibilidad de influir sobre el tamaño de la población. Se ha creído que cualquier proyecto convencional de inversión que promueve desarrollo económico influye positivamente sobre el descenso de la natalidad lo cual a corto plazo no es forzosamente cierto. Por consiguiente, tanto en el ámbito nacional como internacional se está llegando al convencimiento de que es necesario influir directamente sobre la natalidad. De este análisis preliminar se desprende que el país requiere dentro del marco de una estrategia general de desarrollo la formulación y ejecución de una política demográfica. Para lograr este objetivo es importante que el proceso de asignación de recursos contemple como una alternativa de inversión los programas de planificación familiar. Así la asignación de recursos a estos programas debe tener en cuenta los mismos principios utilizados en los análisis de decisión de inversiones en otros campos. Es decir, se justifica dirigir recursos a dichos programas en la medida en que sus análisis beneficiosos muestren que su rentabilidad social es mayor que la de otras inversiones alternativas. Es necesario por tanto romper el efecto del círculo vicioso por medio de un largo y sostenido esfuerzo para elevar el ingreso y los niveles educacionales de la masa de la gente a fin de proveer una motivación adecuada para tener familias más pequeñas". ( $E l$ Tiempo, 1972, 8 de junio, p.10) ${ }^{14}$.

La política de Lleras en su mandato (1966-1970) y la posición que asumió a lo largo de la década del setenta con relación al tema de la familia, fue plasmada en la Declaración de los Jefes de Estado (1967) presentada a la ONU. En ella, a partir de una concepción clara de un Estado liberal y laico, se manifestó que la oportunidad de decidir el número de hijos debía ser un derecho humano fundamental - ejercido mediante el incremento del nivel educativo con el fin de lograr una responsa-

14 "Reducir crecimiento de población pide Lleras". El Tiempo, 8 de junio de 1972, p. 10. 
bilidad mayor en la pareja. En consecuencia, se convirtió en una responsabilidad del Estado laico hacer asequible los servicios médicos necesarios con el fin de asegurar una atención médica correcta que garantizara el respeto debido a la conciencia de los solicitantes.

Con base en lo anterior puede concluirse que la discusión en torno a la planificación familiar, especialmente al uso de la píldora, se constituyó en una práctica social naturalizada de forma paulatina en la primera mitad de los años setenta en Colombia. La preocupación del Estado frente a la alta tasa de crecimiento anual de la población y su relación con la política económica y social - aspecto que se evidencia en el escenario de lo público- hizo parte de la constitución de una política en torno al tema familiar. Así mismo, el discurso concerniente al tamaño de la familia y la decisión autónoma de la pareja en ésta cuestión (además de la aparición de instituciones sociales especializadas en el tema - Profamilia, Encuesta Nacional de Fertilidad de 1969- y su impacto para comienzos de la década del setenta), son los precedentes de un nuevo tipo de familia que se instalaría a partir de ésta coyuntura histórica. En general, el discurso de la planificación familiar, cimentado en la Colombia de los años setenta, facilitó las condiciones de emergencia para un nuevo rol de la mujer, la transformación social de la familia y la constitución de un nuevo tipo de infancia.

\section{Nuevo rol de la mujer}

La planificación familiar, sumada a los aportes desarrollados por la biotecnología y la farmacología, representó un papel fundamental para la materialización de un nuevo rol de la mujer en la sociedad moderna; además de separar el placer sexual de su función reproductiva, logró insertarla en el escenario laboral - aspecto trascendental en su proceso de emancipación como sujeto. Ésta situación, por lo demás, incidió en la nueva estructura de la familia y en las nuevas prácticas de socialización y educación que para ésta época vivía la infancia en el interior del nuevo núcleo familiar.
Para finales de los años sesenta se hacía claro que:

Las madres que salen a trabajar, generalmente no pueden conseguir una persona adecuada para cuidar a sus hijos pequeños... La falta de cuidado materno causa en el niño problemas de todo orden, especialmente de orden sociológico, los cuales han sido ampliamente estudiados en todas partes del mundo incluyendo nuestro país. Esta carencia de afecto y atención por parte de la madre es más perjudicial cuando el niño es muy pequeño y cuando se prolonga por periodos largos de tiempo ${ }^{15}(E l$ Tiempo, 1968, 5 de julio, p. 12).

Planteado así el problema de la incorporación de la mujer al escenario laboral, la sociedad pudo optar por dos opciones: buscar la manera para que la madre no saliera del hogar y no descuidara a sus hijos (lo cual no siempre era posible) o crear los medios necesarios para proteger al niño mientras su madre trabajaba. Entre tanto, la individualización del niño por medio de disciplinas como la psicología y la pedagogía, aportaron de manera sustancial a la solución. Éstas dos disciplinas, mediante el discurso del cuidado y la educación del niño — ubicado más allá de la frontera que establece la familia-, generaron las condiciones suficientes para librar a la mujer de las obligaciones domésticas.

Entonces, la psicopedagogía se convirtió en uno de los resultados directos de la incorporación masiva de la mujer al escenario laboral. Desde esta perspectiva:

De acuerdo con la edad de los hijos se pueden organizar dos tipos de entidades. Salacunas para los lactantes y guarderías y jardines infantiles asistenciales para los mayorcitos. Las investigaciones del periodo permiten observar que los jardines infantiles asistenciales, cuando están bien organizados, no sólo permiten brindar una protección a los menores, sino que pueden evitar las deficiencias de orden nutricional, $\tan$ frecuentes en los niños que viven en los medios pobres. Además ayuda a

15 "Madres que trabajan". El Tiempo, 5 de julio de 1968, p.12. 
su desarrollo psicológico, brindándoles entretenimiento y experiencias que el bajo medio intelectual en que viven no se los podría facilitar" (El Tiempo, 1968, 5 de julio, p. 12) ${ }^{16}$.

En la sociedad colombiana de principios de los años setenta, los hombres - sobre todo los menores de 30 años - tuvieron que aceptar el hecho de que los roles familiares habían cambiado y que, por ende, tenían que hacerse partícipes de la crianza de sus hijos y de los oficios caseros. Lo anterior fue evidente en la modificación profunda del equilibrio tradicional de la pareja y en la emancipación de la mujer respecto a la necesidad que tenían las familias de recibir ingresos extras de la mano de obra femenina - aspecto que obligaba a los hombres a equilibrar las funciones en el hogar.

Por supuesto, éste hecho implicó una reorganización de los roles en la familia y, en consecuencia, en Colombia surgieron ideas que transformaron las reglas tradicionales de la vida entre los dos sexos. Frente a esta nueva situación, "el gobierno ha tomado la iniciativa y dicta leyes que reglamentan el arte de vivir en pareja, de la familia en general. Aún así las mujeres siguen siendo objeto de profundas discriminaciones sociales lo que da motivo para pensar que la mujer se convierta en una fuerza explosiva que se levante contra la sociedad dominada por los hombres"17 (El Tiempo, 1970, 21 de mayo, p. 22).

Además de su nuevo rol como trabajadora en la industria y en la economía, la mujer se convirtió en la principal educadora formal de la infancia moderna; inclusive, se especializó en una práctica y en un discurso de una labor que comenzó a ser remunerada para algunas de ellas, ya fuera como pedagogas o trabajadoras sociales. La Asociación Colombiana para el Estudio Científico de la Población, fundada en 1964, cuya Directora Ejecutiva en ese entonces era Isabel Carrasco de Gómez, conformó un grupo de profesionales de distintas disciplinas especializadas en el tema. Dicha institución, entre

\footnotetext{
16 Ibíd.

17 "Hoy ellos cambian los pañales y lavan los platos". El Tiempo, 21 de mayo de 1970, p. 22.
}

el 16 y 27 de agosto de 1971, dictó en Bogotá el Primer Curso Latinoamericano en Capacitación para Líderes Femeninas, el cual contó con la participación de representantes de Colombia, Bolivia, Costa Rica, Guatemala, Honduras, Nicaragua, Panamá, Perú, El Salvador y Venezuela. En ésta actividad se reconoció que "si bien la familia es la primera célula de toda la sociedad y que la formación básica del hombre ha de ser recibida en su seno, la mujer es el núcleo de esa célula, de ahí la importancia de tener en cuenta a la gran mayoría de mujeres que han de ser educadoras y formadoras, que constituyen grandes valores humanos desperdiciados por falta de oportunidades, por la discriminación de que ha sido objeto y subestimación propia"18 (El Tiempo, 1971, 12 de agosto, p. 19).

Éste contexto garantizó un ambiente de emancipación para la mujer que, liberada del yugo patriarcal, vivía una nueva revolución en la época moderna. Miles de mujeres se fueron concientizando de que su papel como personas no podía reducirse simplemente al ideal de ser madres de familia; lo cual tenía un valor pero no las definía como nuevos sujetos. "Insatisfechos los movimientos feministas hacen su aparición en diferentes países tratando precisamente de definir el papel que como persona le corresponde a la mujer, evidenciando dos tipos de explotación: de tipo sexual y de tipo económico"19 (El Tiempo, 1970, 10 de mayo, p. 70).

Hacia mediados de la década del setenta las madres jóvenes, particularmente las que trabajaban y tenían una profesión, se enfrentaron a una alternativa difícil frente al nacimiento de sus hijos: por un lado, podían consagrarse por entero a su crianza y educación (sacrificando sus actividades) o, por el contrario, debían continuar en su trabajo absteniéndose de pasar algunas horas cerca del bebé. Así pues, las costumbres, las tradiciones y la forma cómo había sido educada la sociedad colombiana hasta entonces, influyeron de manera determinante en éste complejo de culpa inicial en las madres jóvenes.

18 "Curso de promoción familiar". El Tiempo, 12 de agosto de 1971, p. 19.

19 "Madres sí, mujeres no". El Tiempo, 10 de mayo de 1970, p. 5. 
En términos generales, desarrollando un balance para la época, hubo mucho de cierto en las prácticas tradicionales de crianza porque "precisamente en los primeros doce meses se inicia entre el niño y el adulto un diálogo destinado a durar toda la vida, con diferentes enfoque según las distintas edades. Y es la madre quien por instinto debería conocer mejor que nadie a su propia criatura quien puede ser capaz de llevar a cabo esta tarea. Por estas razones algunos sicólogos consideran insustituible la afectuosa presencia de la madre al lado del niño" ${ }^{20}$ (El Tiempo, 1975, 14 de febrero, p. 1B).

Por su parte, en la práctica, muchos pediatras opinaron que la mujer podía alejarse de su hijo durante varias horas del día sin que por ello fuera a sufrir graves daños en su desarrollo o en su equilibrio nervioso; lo que importaba realmente era que el niño fuese confiado a una persona capaz de establecer con él una relación serena basada en el buen entendimiento. Se estableció entonces que el afecto y la atención a los pequeños no era cuestión de cantidad sino de calidad. La madre podía atender las obligaciones de su profesión o de su trabajo si a cambio, durante su tiempo libre, sabía acercarse a sus hijos con serenidad y comprensión; interesándose, así mismo, por sus problemas y necesidades.

De ésta forma, tales preocupaciones eran relegadas pues:

Numerosos sicólogos han demostrado que un niño crece mejor y desarrolla toda su personalidad si en la infancia recibe alegrías y sinsabores, en proporciones iguales y mejor aún si estos prevalecen en una mínima parte. Ante la situación de la mamá trabajadora, la solución más recomendada por sicólogos y pedagogos infantiles es la de llevar al niño a una guardería. Las hay que reciben a los niños desde los dos meses hasta los cuatro años, es decir cuando comienza la educación escolar. Allí los pequeños reciben atención profesional, que garantiza la tranquilidad de

20 "Cuando la mamá trabaja y el hijo queda en casa". El Tiempo, 14 de febrero de 1975, p. 1 B. las madres ${ }^{21}$ (El Tiempo, 1975, 14 de febrero, p. 1B).

El nuevo rol de la mujer estableció prácticas modernas de crianza que fueron reconocidas por la sociedad y validadas por diferentes disciplinas. Dentro de ellas, la calidad del tiempo dedicado a los hijos fue una preocupación que empezó a discutirse desde diferentes perspectivas pedagógicas, psicológicas y pediátricas; demostrando, a la vez, la necesidad de reflexionar en torno al tema de la primera infancia — en la que se establece una nueva relación entre la familia y la escuela. Éstas instituciones, conformadas en el país a lo largo de la década del setenta, contaron con el apoyo del Estado y establecieron una realidad que comprometió a las nuevas prácticas de socialización y educación de la infancia.

El desempeño de la mujer en labores que en otro tiempo eran exclusivas de los hombres no fue catalogado como bueno o malo por gran parte de la opinión pública; en sí mismo, obedeció a variables socioculturales que lo determinaron y eran gestoras de un nuevo tipo de desarrollo. Desde ésta premisa se debía partir, entonces, para analizar la nueva situación de la familia colombiana en el marco de la sociedad en que está inscrita, dependiente y subdesarrollada, haciendo parte de una serie de problemas socioeconómicos que, directa o indirectamente, afectó el núcleo social base; es decir, la familia.

En el desarrollo de ésta coyuntura, la institución familiar tuvo que afrontar una de las crisis más agudas provocadas a causa del cambio social continuo - acelerado especialmente en los últimos años. Para el sociólogo norteamericano William J. Goode:

No por ello se puede hablar de la muerte de la familia. La gente que está experimentando nuevas formas de vida no es numerosa, en la mayoría siguen viviendo en familias de modo muy tradicional y, considerando esto, como la mejor solución para sus necesidades tanto bajo el capitalismo como el socialismo. Aunque

21 Ibíd.

REVISTA CIENTÍFICA / ENERO -DICIEMBRE DE 2010 / No. 12 / BOGOTÁ, D.C. 
el sistema de familia no cambia con rapidez la tendencia va hacia la reducción de la familia y hacia una mayor libertad de los hijos con relación a sus padres en lo que concierne a la lección de trabajo o carrera y en cuanto a la libertad sexual. Por lo demás para obtener un alto nivel de producción se necesita pasar unas horas del día en una atmósfera sentimental y desarrollar relaciones amorosas que sólo se pueden colocar fuera del marco burocrático y tecnocrático" 22 . (El Tiempo, 1976, 6 de mayo, p. 3E).

Por lo tanto, el nuevo rol de la mujer generó polémica y discusión, aspecto que aún se reflexionaba a finales de los años setenta. A su vez, para algunos sectores conservadores de la sociedad ocasionaba un cierto grado de tristeza ver

Cómo las mujeres muestran indiferencia por la maternidad; no sólo evitan los hijos, sino que quieren no ser madres. Las jóvenes de hoy no tienen el interés de antaño por ser madres. Los tiempos han cambiado y cómo han traído cosas buenas, también han dejado de lado los valores, hoy la mujer se decide a estudiar hasta ser profesional, impidiéndole ser madre, restándoles tiempo a sus hijos e impidiéndoles educar, formar, cuidar, proteger y defender a los niños que deben ser su preocupación. ${ }^{23}$ (El Tiempo, 1978, 11 de mayo, p. 3D).

Entre tanto, fundamentado en una visión más liberada de la vida, existió otro sector de la sociedad que destacaba cómo para comienzos de los años ochenta se estaba viviendo en el mundo un cambio social, económico, político y tecnológico. Ésta transformación, cada día más veloz, influyó notablemente en las costumbres y en los hábitos de la vida familiar.

Casi se puede afirmar que ha cambiado la gente. El sexo femenino entre otras cosas ha recibido gran influencia de cambio. Se afirma que una madre moderna no tenía el más remoto parecido con las abuelas. Las mujeres de este tiempo son diferentes

22 "La familia no morirá". El Tiempo, 6 de mayo de 1976, p. $3 \mathrm{E}$.

23 “En decadencia el instituto maternal?”. El Tiempo, $11 \mathrm{de}$ mayo de 1978, p. 3D. a las de hace 20 ó 30 años en todo sentido, frente a la vida, la sociedad y el trabajo, también frente al matrimonio, la maternidad, el amor o al sexo. Ellas consideran que deben participar en el campo laboral para así poder optar económicamente $\mathrm{y}$ adquirir independencia asumiendo que pueden opinar, actuar y discutir libremente. Por ejemplo, en el mercado laboral los empresarios contratan un mayor número de mujeres aduciendo ciertas ventajas con respecto a los hombres: consagración, seriedad, responsabilidad, menores gastos, mayor organización y menor consumo de alcohol" 24 . (El Tiempo, 1982, 5 de mayo, p. 3D).

La incorporación masiva de las mujeres al escenario laboral y al trabajo remunerado supuso menoscabar la legitimidad en la familia tradicional y, en especial, del padre como único proveedor. Así mismo, la crisis de la familia tradicional influyó significativamente en la transformación de la identidad, sexualidad, naturalización y socialización de los nuevos sujetos.

En cuanto al ámbito intelectual, en 1982 la filósofa Elizabeth Badnier escribió La historia del amor maternal, obra donde evidenció un balance histórico del instintito materno y lo describió como un mito inventado por Occidente para sojuzgar a las mujeres. En consecuencia, ésta percepción la convirtió en la mujer más odiada de Francia y se llevaron cabo varios debates televisivos con fin de cuestionar el contenido del libro. Por lo demás, a la escritora le llovieron calificativos como el de $<<$ monstruo $>>$, $<<$ anormal $>>,<<$ degenerada $>>$ $\mathrm{y}<<$ asquerosa $>>$; afirmó, además, que tal instinto era un fenómeno cultural y que no existía ninguna prueba para demostrar que ser madre era algo innato. Incluso, el texto contenía un balance corto acerca del desprecio hacia los niños y los casos de infanticidio y abandono entre los siglos XVI y XVIII — señalando la publicación de libros sobre esta temática. "Ahora surgen diversas opiniones sobre elegir o no ser madre admitiendo dichas mujeres que el llamado instinto maternal no es otra de las fórmulas mágicas e irracionales con las que

24 "Las madres modernas". El Tiempo, 5 de mayo de 1982, p. $3 \mathrm{D}$. 
la sociedad ha querido someter a la mujer y ante las cuales ella no puede reaccionar porque se convierte en un ser desnaturalizado y monstruoso" 25 (Carrusel, 1982, 7 de mayo, p. 13).

Discursos como el anterior fueron menoscabando paulatinamente el tipo de familia tradicional que aún existía en ciertas zonas del país. Las mujeres, en el escenario urbano a mediados de los años ochenta, optaron por tener un promedio de dos hijos como máximo. "Las mujeres sacrificadas con más de diez hijos pasaron de moda como los pañales de tela, los teteros de vidrio y los corrales de madera. A una madre moderna el tiempo no le alcanza para más de dos hijos porque la oficina le absorbe todo el tiempo. En la actualidad se está dando paso a las madres ejecutivas y otras trabajadoras, con mentalidad especial de pañal desechable"26 (El Tiempo, 1984, 12 de mayo, p. 1E).

Hacia 1985, la familia colombiana se acomodó al devenir de la cultura y la sociedad; así mismo, los cambios más destacados se relacionaron con sus funciones frente a la comunidad, la vida de pareja y las relaciones filiales. Entonces, la pareja comenzó a ser asumida como un sistema experimental en el que se ponía en juego el ajuste de las personalidades libres para llegar con mayor madurez al matrimonio - si se llegaba - y así evitar el conflicto de la ruptura nupcial. La relación esporádica acercó a la pareja con instancias de la atracción afectiva, manteniendo cada uno su residencia y estatus de soltería; no se creaban lazos sociales y se satisfacía la necesidad biológica amorosa, concediendo un tipo de libertad importante de vinculación de sus miembros.

Virginia Gutiérrez de Pineda, especialista en la familia colombiana, afirma que:

El madre solterismo como consecuencia de las alternativas domésticas anteriores genera una familia incompleta en la que la falta de figura paterna genera una inestabilidad familiar. La

25 . "Rebelión contra la maternidad". El Tiempo, 7 de mayo de 1982, revista Carrusel.

26 . "Mamá, los hijos pasaron de moda". El Tiempo, 12 de mayo de 1984, p. 1E. educación masiva ha sido un factor influyente en los cambios de estructura familiar, porque el individuo entra sólo a competir por ubicarse, su grupo doméstico ya no puede acompañarlo. Para poder ascender las jerarquías sociales no puede arrastrar consigo a todos los suyos, cada persona lucha sola, se ubica sola, responde por sí misma y sólo recibe la gratificación de sus esfuerzos. Y en la mujer este avance intelectual también es determinante, porque ahora existe una lucha de poder entre el hombre y la mujer ${ }^{27}$ (El Tiempo, 1984, 13 de junio, p. 4C).

La anteriores condiciones generaron la posibilidad para que emergieran nuevas prácticas y discursos de carácter psicológico, pedagógico y pediátrico que posibilitaran otro tipo de educación para la primera infancia. La mujer trabajadora, como expresión de emancipación, transformaría de manera implícita las relaciones heterosexuales, de carácter tradicional, que se establecían en la familia moderna. Tal aspecto no sólo incidiría en la identidad sexual y personalidad de las nuevas generaciones, sino que establecería en la familia moderna una serie de pautas contemporáneas de crianza y socialización.

\section{El niño en el primer plano}

En la década de los años setenta, el niño se encontraba en el primer plano de las preocupaciones sociales; exigía cariño, atención, cuidado, compañía, estimulación y juegos. No obstante, como a todo sujeto nuevo que emerge, se desarrollaron importantes esfuerzos por individualizarlo a partir de los discursos de las prácticas de crianza y de la socialización mediante diferentes disciplinas (psicología, pediatría, psicoanálisis, etc.) con fin de reconocerlo como sujeto nuevo.

En éste sentido, el psicólogo francés René Le Senne desarrolló un estudio a inicios de los años setenta que se dio a conocer en Colombia -con el cual quiso determinar ciertos factores dominantes en

27 "La familia, crisis o cambio". El Tiempo, 13 de junio de 1984, p. 4C.

REVISTA CIENTÍFICA / ENERO -DICIEMBRE DE 2010 / No. 12 / BOGOTÁ, D.C. 
los diferentes carácteres de los niños. Recopilando la información, el psicólogo los catalogó en cinco grandes grupos teniendo en cuenta tres elementos básicos de todo ser humano: la emotividad (o no emotividad), la actividad (o no actividad) y la forma de reaccionar ante los pensamientos y acontecimientos - puede ser inmediata o retardada. Para éste especialista, la tarea de conocer bien a sus hijos y hacer de ellos seres responsables y equilibrados le corresponde exclusivamente a los padres. "En el informe del dr. Senne se clasifican cinco tipologías de niño con una propuesta particular de tratamiento y educación: el niño nervioso, el niño sentimental, el niño colérico, el niño apasionado y el niño apático"28 (El Tiempo, 1975, 16 de abirl, p. 1B).

Por su parte, la pediatría desarrolló un esfuerzo importante por individualizar al niño -en el contexto familiar, antes que nada. Para el especialista Jaroslav Koch, el hombre podía dar más de sí teniendo en cuenta lo que hasta en ese momento se le había permitido. La escuela ya no podía proporcionar más y por eso la sicología buscaba en el hombre sus reservas en la edad lactante y en la primera infancia. Este psicólogo, que siempre estuvo atraído por el estudio de los más pequeños, se ocupó de la problemática de la educación en la sala cuna y escribió un libro sobre $<<$ casas cunas $>>$ en el Manual de sicología y pedagogía infantil -difundido en Praga. En éste se aludía a la estimulación temprana y el acompañamiento de la madre en los dos primeros años de vida (aspecto que incidió para aumentar en su país a dos años la licencia de maternidad para madres empleadas). El desarrollo motriz de la mano del niño, como ejercicio fundamental para el desarrollo de su inteligencia, fue una de las observaciones que dio a conocer este psicólogo durante los años sesenta. Para la psicología y la pediatría, en ese entonces, el mundo demandaba que la gente fuera capaz y rindiera mucho más desde todos los puntos de vista, aprovechando las reservas que se encontraban en la edad de la primera infancia. "El mundo trae al niño una cantidad de posibilidades de desarrollo y éstas se despliegan hasta cierto periodo en la edad del niño en condiciones y

28 "Cada niño es un mundo, no quiera educarlos igual". El Tiempo, 16 de abril de 1975, p. 1B. con impulsos adecuados. Luego las posibilidades se desvanecen. Los impulsos dados en la edad más temprana ejercen una influencia inmensa sobre todo el desarrollo el hombre y predestinan todo el desarrollo consecutivo en la edad posterior" ${ }^{29}$ ( $E l$ Tiempo, 1975, 4 de diciembre, p. 1B).

En sus estudios, Jaroslav Koch demostró que aquellos niños que en su infancia habían contado con suficientes posibilidades de jugar y de observar su alrededor, desarrollaban ejercicios valiosos que los preparaban para el aprendizaje de la escritura y la lectura -asimilándolos en menor tiempo. Éstos niños llegaban a la escuela con buenas bases para iniciar su instrucción; sabían asociar las ideas, relacionarlas y compararlas, pues habían aprendido a coordinar sus propios movimientos (facilitadad para el manejo del lápiz) y ahora se destacaban en el análisis de las palabras escritas y en el descubrimiento de ciertas letras repetidas.

De acuerdo con la pediatría, la pobreza de ideas era consecuencia de una mala preparación en la temprana infancia. El niño que presenta dificultades para redactar las composiciones escolares no está acostumbrado a observar la realidad que lo rodea y nunca se ha detenido atentamente a considerar la realidad en el tiempo y el espacio -que se logra mediante figuras de libros, tarjetas ilustradas, imágenes de televisión o el cine.

Estos ejemplos ilustran a los padres en dos aspectos, el niño que llega a la escuela con una personalidad formada ya en gran parte y los resultados escolares a los que acostumbramos a limitar nuestra atención, son producto de procesos largos e inadvertidos a los que deberíamos prestar más interés. Si por un lado la diversión absorbe el tiempo, por el otro, satisface exigencias fundamentales de los niños, y por lo tanto, les permite rendir mucho más

29 "Lactancia. Secretos de la educación en el primer año de vida”. El Tiempo, 4 de diciembre de 1975, p. 1.B. El diario El Tiempo especificó que Jaroslav Koch es un psicólogo nacido en una familia checa residente en Viena. Hizo estudios en la Facultad de Filosofía de esa ciudad y dio clase checas de Comenio en Viena. Su disertación se titulaba: "Comportamiento social de niños de 10 a 14 años de edad en trabajo y juego colectivo". 
en el estudio. Es decir, que elegida bien calidad y cantidad, las diversiones favorecen en gran medida la maduración de los hijos y los hace más responsables respecto a los diferentes aspectos de su vida" ${ }^{30}$ (El Tiempo, 1975, 4 de diciembre, p. 1B).

Las reflexiones desarrolladas desde éstos puntos de vista apuntaron a reconocer al niño moderno como sujeto desde, por los menos, 1977. La emergencia de éste $<<$ nuevo niño $>>$ la retrata el investigador Haim Gruspum, Director del Instituto Sedes Sapientiae de Sao Pablo; en una investigación referente a la influencia del niño en la sociedad manifiesta que:

Hasta hoy todo el mundo reconocía a ojos cerrados que la sociedad influye de manera significativa en el niño, pero jamás nadie se cuestionó si era posible obtener la respuesta contraria. El niño también influye en la sociedad, dejó de ser pasivo y el gran cambio de la sociedad moderna es exactamente esa participación activa del niño. El cambio ocurrió en el momento en el que el niño se convirtió en el eje de la familia. Hace cincuenta años el padre centralizaba el poder de la familia. Hoy todo está centralizado en el niño, antiguamente el niño no contaba en la familia y eso se reflejaba en la sociedad" ${ }^{31}$ ( $E l$ Tiempo, 1977, 6 de junio, p. 17).

Por otro lado, la colombiana Marisa Uribe Hurtado se mostró sorprendida al descubrir que la niñez, sólo hasta comienzos del siglo XX -como concepto genérico-, dejó de ser la categoría que cobijaba a los niños propiamente dichos, los retardados mentales y los salvajes de cualquier edad.

El siglo XVII definió a los niños como débiles e indefensas criaturas de Dios, a las que había que proteger, rehacer y poner en orden, manteniendo a toda costa su inocencia, a tiempo que estimulaba su intelecto. Como dato aún más curioso, el concepto de niñez, se desco-

\footnotetext{
30 Ibíd.

31 “Una nueva teoría. ¿Es necesario que el niño sufra?". El Tiempo, 6 de junio de 1977, 17.
}

noció completamente durante la Edad Media. La niñez y la adolescencia se fundían en una misma época cronológica, se carecía de las diferencias existentes entre los mundos del niño y del adulto. La niñez simplemente era una etapa sin importancia alguna, en el desarrollo del individuo acerca del cual no había necesidad de profundizar en forma alguna $y$, en segundo lugar, puesto que lo más probable era que el pequeño muriera32. (El Tiempo, 1977, 1 de agosto, p. última $B$ ).

Así mismo, para Hurtado, el origen de la sensibilización se debe al auge de la narrativa y la pintura en torno a la infancia de Jesús:

La 'Sagrada Infancia', impregnó e imprimió el concepto de niñez por primera vez en la sociedad de aquellos tiempos. El niño fue visto de manera diferente del adulto, al describirse el detalle de sus adelantos año por año y al relatarse anécdotas de sus primeros años de vida, a pesar de toda esa influencia religiosa todavía se concebía al niño como persona débil, debilidad encadenada estrechamente con la inocencia de Jesús y, por consiguiente, con la niñez como estado. La idea de inocencia de los niños produjo como resultado dos tipos de comportamiento. De una parte era necesario proteger al infante de las impurezas del medio y por otra debía forzársele a que desarrollara un fuerte carácter y un razonamiento competente. Ya para el siglo XIX la condición de la niñez cambia en forma constante y radical. Surgen grandes escuelas de sicología y psiquiatría y empieza a darse nombre y sentido a las primeras etapas cronológicas del hombre. Ya no se habla de niñez, sino de primera infancia, pubertad, adolescencia y juventud33. (El Tiempo, 1977, 1 de agosto, p. última B).

Ésta nueva disciplina -la histórica-, que se sumó al proceso de decantación e individualización

32 "El chantaje del afecto al niño". El Tiempo, 1 de agosto de 1977, última B.

33 Ibíd.

REVISTA CIENTÍFICA / ENERO -DICIEMBRE DE 2010 / No. 12 / BOGOTÁ, D.C. 
discursiva del niño, fue reafirmada por la postura pedagógica que, en un inicio, se había constituido también desde el discurso del cuidado de Jean Jaques Rousseau -comenzaría a dejar una impronta en las reflexiones sobre éste sujeto. La infancia, entonces, podía ser pensada desde dos ángulos: en términos de cuidado y protección o, por su parte, de abandono. Así pues, mediante las lecturas de Rousseau, que se popularizaron en el país a comienzos de la década del ochenta, el instinto paterno pudo inhibirse o exagerarse; el animal abandona la cría rápidamente pero el hombre prolonga la protección por periodos largos debido a la naturaleza creadora y a la potencialidad en desarrollo del ser humano.

Entre tanto, el psiquiatra colombiano Eduardo Jones, en 1981, dio a conocer que un alto porcentaje de los niños colombianos presentaba síntomas de ansiedad. Advirtió, además, que los problemas habían sido clasificados de leves a graves y se presentaban específicamente en la población con contrariedades como el abandono y economía precaria: "efectivamente, el 70\% de los niños colombianos presentan manifestaciones cíclicas, disturbios emocionales que se pueden clasificar de leves a graves según el concepto del pediatra" ${ }^{34}$. Así mismo, señaló que los problemas más graves pertenecen a la población indigente debido a la inestabilidad familiar, al abandono de la madre y la baja estimulación precoz, sensorial y perceptual; hechos que conllevan al retraso en el desarrollo psicomotor del niño, así como a problemas de aprendizaje y afectivos.

Igualmente, advierte que en los hospitales es notorio observar niños pertenecientes a la clase baja que han sido abandonados y ni siquiera presentan reacción de ansiedad, síntoma indicativo de un estado marcado por la depresión y problemas afectivos entre el niño y su madre; es claro entre los niños de la clase media su ansiedad y que, según el psiquiatra infantil, se manifiesta en acciones agresivas como golpear a otros niños, arrebatarles

34 "Depresión: síndrome endémico de la población infantil colombiana”. El Tiempo, 14 de septiembre de 1981, p. 11D. violentamente las cosas o en una hiperactividad motora que, con más sencillez, cobija lo que los psiquiatras llaman niños inquietos.

Por otra parte, Jones asegura que:

Llevarse objetos a la boca, comerse las uñas, falta de concentración y atención, son síntomas de ansiedad. El niño manifiesta sus afectos más que con palabras con actitudes o conductas generalmente motoras debido a la falta de desarrollo en su lenguaje. Otros grados de depresión tienen manifestaciones diversas. La irritabilidad es la más frecuente. Afirma también que el aislamiento del niño en edad escolar al igual que el bajo rendimiento en estudios son expresiones de depresión. La inestabilidad económica y el abandono se presentan como las principales causas del problema. No sólo el abandono físico sino también el abandono emocional del niño ${ }^{35}$ (ibíd.).

La psicoterapia aplicada por el doctor Jones en los niños, a través del juego y el dibujo, dio cuenta de un nuevo esfuerzo disciplinar por individualizar los problemas hepáticos y de neurosis en los más pequeños -tarea que hasta ese entonces se iniciaba en Colombia. En la psicoterapia infantil -explicada por él- siempre se recurrió a una tercera persona paralela al problema y nunca se identificó al niño como personaje de éste; la tercera persona podía ser un animal o el personaje de un cuento mediante el cual el niño expresaba sus fantasías y sentimientos $y$, de ésta forma, empezaba a ser consciente de su problema.

Los niños con potencialidades hicieron parte de éste ejercicio de individualización que, del ámbito científico, se trasladó al escenario social. En éste sentido, se aclaró que 5 de cada 100 niños tenían cualidades excepcionales y se estimó que 2 de cada 600 contaban con un coeficiente intelectual (CI) alto; sin embargo, a la mayoría de ellos nunca se les reconocía su capacidad (por lo menos por parte de sus padres). Ese fue el señalamiento que realizó contra los padres uno de los principales psicólogos educacionales de Gran Bretaña, el doctor Te-

35 Ibíd. 
rry Morgan, quien aseguró, a comienzos de lo años ochenta, que ellos son los últimos en enterase de que tienen un hijo genio.

En efecto, en el mismo informe se reseñaba que los niños superdotados podían parecer descuidados e inclusive desadaptados en el colegio, cuando en realidad sólo intentaban acelerar su trabajo debido a su ansiedad por aprender más.

Hay algunas pocas reglas que los padres podían seguir como guía para conocer el potencial de sus niños entre las edades de cinco a siete años. La lectura, a menudo, es un buen indicador de una inteligencia sobresaliente, no la cantidad sino cuanto se comprende y se recuerda. La habilidad para aprender por la experiencia es otro punto de gran valor, talento para la invención e improvisación. Por lo general, es un niño que desea ser aceptado en el mundo adulto y esto se demuestra con su comportamiento precoz y por su don de mando frente a los demás niños. Si se le da la oportunidad al niño superdotado puede hacer maravillas. Hablarán con mayor fluidez, tendrán un mayor vocabulario y su afán de aprender será mucho más fuerte ${ }^{36}$. (El Tiempo, 1981, 12 de agosto, p. 1E).

\section{Conclusiones}

La individualización del niño, por medio de diversos discursos de carácter social y científico, supuso su reconocimiento como un sujeto que se posicionó en el primer plano durante los años setenta e inicios de la década de los ochenta. En el ámbito social, el carácter del niño, de acuerdo a la emotividad, actividad y reacción ante los acontecimientos, como también las diferentes investigaciones y reflexiones en torno a la estimulación temprana y al papel de un nuevo agente de socialización en su expresión de educación preescolar, así lo dejaron entrever.

36 “Cómo descubrir al niño superdotado?”. El Tiempo, 12 de agosto de 1981, p. 1E.
Así mismo, las iniciativas realizadas por la pediatría con fin de identificar las reservas cognitivas de la primera infancia para explotarlas en beneficio de la personalidad y la identidad del adulto, representan otra expresión de éste reconocimiento. Por lo demás, todos los anteriores esfuerzos derivaron en el reconocimiento del niño como sujeto en el interior de la familia, así como la manifestación de su autonomía -que influyó en la sociedad-, aspecto que evidenció el paso de la infancia moderna a la infancia contemporánea (a la cual se le reconoce como portadora de una subjetividad).

En el ejercicio de individualización del niño, iniciado desde el momento en que la familia empezó a transformarse, la mujer ejerció un nuevo rol en la sociedad y, como consecuencia, se modificaron las prácticas de crianza. Éste proceso estuvo sustentado en un esfuerzo importante de varias disciplinas (psicología, pediatría, psicoanálisis, historia y pedagogía) por enunciar ese nuevo sujeto -denominado infancia- que exigía ser controlado y delimitado a través de un discurso que abarcara un ejercicio de objetivación particular.

\section{Fuentes}

Archivo del diario El Tiempo (1968-1984).

\section{Bibliografía}

- Aries, P. (1987), El niño y la vida familiar en el antiguo régimen, Madrid, Tauros.

- Castells, M. (1999), La era de la información: economía sociedad y cultura, México D.F.. Siglo XXI.

- Deleuze, G. (1987), Foucault, Barcelona, Paidós.

- Elias, N. (1998), La civilización de los padres y otros artículos, Bogotá D.C., Norma.

- Foucault, M. (1976), Historia de la sexualidad. La voluntad de saber, México D.F., Siglo XXI.

REVISTA CIENTÍFICA / ENERO -DICIEMBRE DE 2010 / No. 12 / BOGOTÁ, D.C. 
- Foucault, M. (1991), Microfísica del poder, Madrid, Piqueta.

- Giddens, A. (2004), La transformacón de la inimidad. Sexualidad, amor y erotismo en las sociedades modernas, Madrid, Cátedra.

- Hard, M. y Negri, A. (2002), Imperio, Barcelona, Paidós.
- Quiceno, H. (2007), Sujeto y Subjetividad en Foucault, Bogotá D.C., Inédito.

- Schérer, R. (2005), Foucault, la pedagogía y la educación, Bogotá, D.C., Magisterio.

- Zuluaga, O. (1999), Pedagogía e historia. La historicidad de la pedagogía. La enseñanza un objeto de saber, Bogotá D.C., Anthropos. 